\title{
Modelling and Simulation of Vessel Surgery based on Mass-spring
}

\author{
Da Ren, Yiming Chen, Bojun Lin, Fanjin Zeng, Jiaming Huang and Jinfeng Wang \\ Department of mathematics and informatics, South China Agricultural University, Guangzhou, 510642, China
}

\begin{abstract}
As the technology developing, precision and accuracy required in medical surgery can be realized through virtual-reality technology in computer aided systems, so that it can satisfy with medical experiments and teaching. In surgery simulation on soft tissues, Mass-spring takes the important roles on simulating the surface transformation of tissues. In this article, we established an intelligent simulation platform for surgery of vein in which includes the transformation based on Mass-spring. This platform can provide good human-computer interface and control some simple motions. It is convenient for medical teaching to instruct the operation scene.
\end{abstract}

\section{Introduction}

Medical simulation mainly produce virtual projects by using 3D reconstruction, new Photoelectric display, human-computer interaction, computer graphical technology and medical data visualization. The surgery simulation and visualization can provide an actual and immersed virtual environment for doctors by fusing multiple technologies. It can imitate the operation scenes from visual, audition and tactile angles. Surgery simulation provides strong support on the stages of preoperative planning, Intraoperative assistance and postoperative rehabilitation, in which Geometry modeling and physical modeling are fundamental technologies $[1,2]$.

In the early development, transformation of soft tissues is the key technology in virtual surgery simulation. Transformation modeling includes two kinds of methods: Finite element and Mass-spring model. Finite elements method has good stability and high accuracy. But it is hard to implement and compute. Comparably, Mass-spring is easier and has little computation. Mass-spring has been applied into many fields, such as transformation of soft tissues, cloth deformation and water surface [3]. Mollemans proposed Mass-spring model based on tetrahedron [4], in which each vertex corresponds to each particle and each edge corresponds to each spring. This model was close to elastic structure. In this article, our group proposed an intelligent platform for simulating the vein surgery based on Mass-spring. In traditional method, the force coefficient is set manually. In our model, we tried to tune the parameters using optimal algorithms.

The article is structured as follows. The model based on Mass-spring will be presented in section 2 . Then, a virtual surgery simulation platform will be introduced in the next section. In final section, analysis and conclusions will be drawn.

\section{Physical Model of Vein based on Mass-spring}

\subsection{Mass-spring model}

Mass-spring can be treated as a piece of cloth like a new container of many masses. Each point has own quality without size. It is just the point mathematically and all points are distributed evenly. One spring connects two points and one point can connect multiple springs. These springs are linear and follow to Hooke's law. The topology structure is shown as Figure 1. 


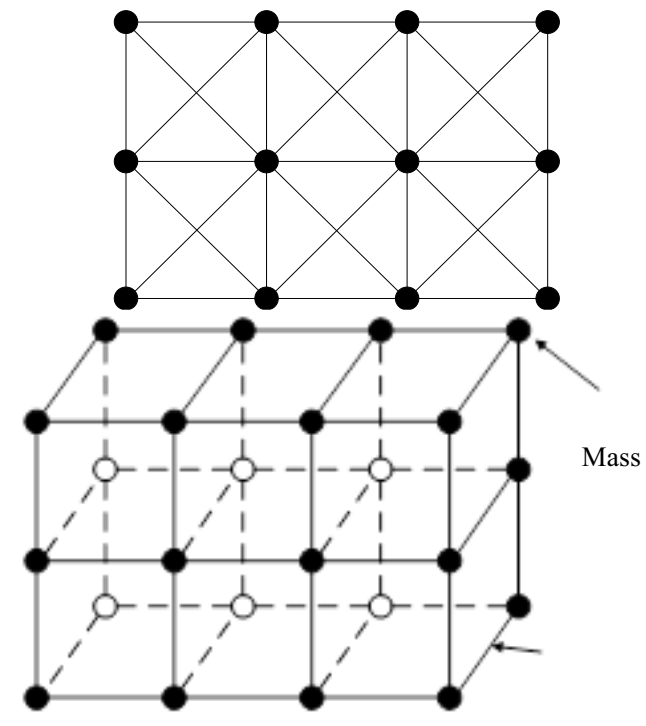

Spring

Figure 1. Topology structure of Mass-spring

Mass-spring includes twist spring, structure spring and tension spring as shown in figure 2. Twist spring means to the spring between diagonal vertices which can prevent tissues twisting abnormally. Structure spring can fix the whole tissue by connecting adjacent nodes. Tension spring connect every two points in order to help folding smoothly. Displacement of points is affected by many factors $[5,6]$. One part is outer force which maybe single gravity, wind power, force from users or resultant of forces. The other means the elastic forces coming from the internal of Mass-spring. The affection to mass-point is the joint forces of external and internal ones. Accelerated velocity of some mass-points can be computed $[7,8]$.

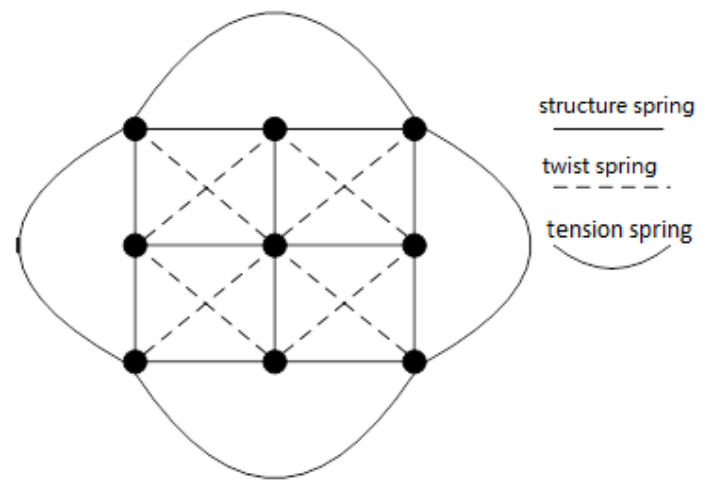

Figure 2. Fundamental structure of Mass-spring

In this article, we consider vessel as soft tissue surface model with uniform mass distribution, which can be partitioned equably into grids. We subdivided the grids further for improving accuracy and adopted grid topology structure as Figure 1 for enhancing real time performance in our model.

\subsection{Setting parameters}

Given that elastic modulus $E$, the corresponding spring constant $k$ can be induced by $k=E \frac{A}{H}$, in which $A$ is the cross-sectional area and $H$ is the height. So we can obtain $k=E \frac{V_{T}}{L^{2}}$, in which $V_{T}$ equals to the third of total volume of tetrahedrons which contain some points; $L$ is the distance between the vertices of tetrahedron and this point. Based on these inferences, all parameters can be placed into the mechanical equation of Mass-spring. Let mass-point be $i$, each force on $i$ would be decomposed respectively along $\mathrm{x}$-axis, $\mathrm{y}$-axis and $\mathrm{z}$-axis. We take $\mathrm{x}$-axis for example to explain as follows.

$$
F_{i j}=\left\{\begin{array}{l}
\frac{x_{j}-x_{i}}{\left\|x_{j}-x_{i}\right\|}\left(k_{i}\left(\left\|x_{j}-x_{i}\right\|\right)-L_{i j}\right), \Delta x_{i}<\Delta x_{i c} \\
\frac{x_{j}-x_{i}}{\left\|x_{j}-x_{i}\right\|}\left(k_{1}\left\|\Delta x_{c}+k_{2}\right\|\left(\left\|x_{j}-x_{i}\right\|-L_{i j}-\left\|\Delta x_{c}\right\|\|\|\right), \Delta x_{i} \geq \Delta x_{i c}\right.
\end{array}\right.
$$

in which $x_{i}, x_{j}$ correspond to vectors of vertices on spring, $L_{i j}$ is the static distance between two points.

Supposed that there are $N$ tetrahedrons related to one point $i$, the volume of these tetrahedrons is $V_{i}$, the mass and volume of vessel is $M$ and $V$, so the mass of this point can be computed as:

$$
M_{i}=M \times \frac{V_{i}}{V \times N \times 3}
$$

Then, we can attribute the mass of vessel into each point uniformly, which means the mass of each point is confirmed.

\subsection{Force analysis}

For any point in Mass-spring model, its dynamic characteristics can be represented as $m_{i} \ddot{x}_{i}+\beta_{i} \dot{x}_{i}+\sum_{j}^{n} F_{i j}+F_{e x}=0 \quad$ according to Newton principle, in which $m_{i}$ is the mass of point $i, x_{i}$ is the coordinate vector, $\beta$ is the damped coefficient of spring 
and $\sum_{j}^{n} F_{i j}$ is the sum of forces affected by other points. When one point was stressed, the deformation depends on the external force and acting forces from other points.

\section{Virtual surgery simulation platform in VTK}

We established an intelligent platform based on VTK (Visualization Toolkit) using Visual $\mathrm{C}++$. The vessel model was induced into system and each point can be extracted and incorporated in to Mass-spring model. After constructing the model, when the medical instruments like needle collide to the vessel, we need produce force on the touching point and make it move in a certain domain. Under Mass-spring action, the movement of touching point presents a satisfied visualization with high precision and accuracy. The flowchart of building platform is shown as Figure 3.

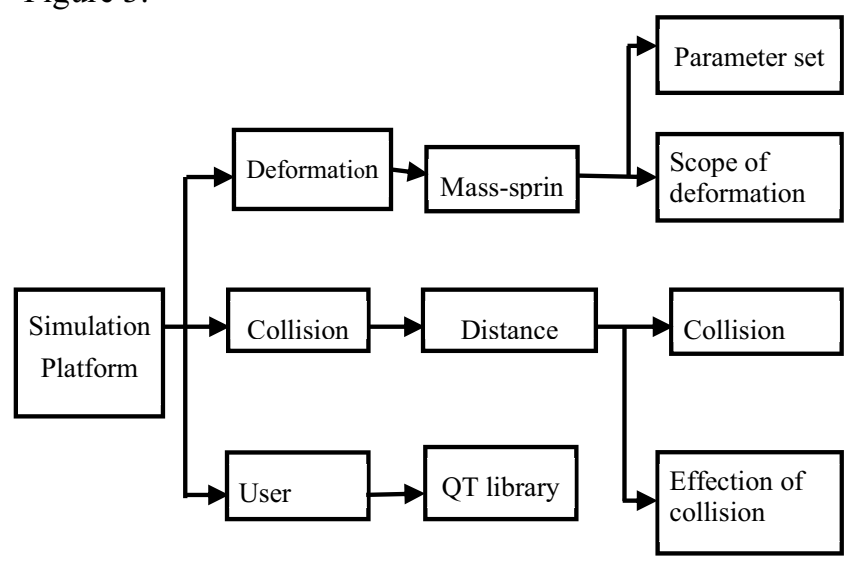

Figure 3. The flowchart of intelligent platform

We adopted classical programming structure for Mass-spring model in constructing model process. The location of point was described by coordinates and springs were established between points. When point was stressed to move, one triggered function can correct the location of the point according to the Mass-spring theory. For describing elastic behavior better, we added the body spring into system, which can keep tissue out of excessive deformation and ensure the model return to the original status. In our platform, we take needle for example to show the simulation process. The effect of this system is realistic enough and acceptable for operators. Several screenshots were selected to show as figure 4(a) and (b).

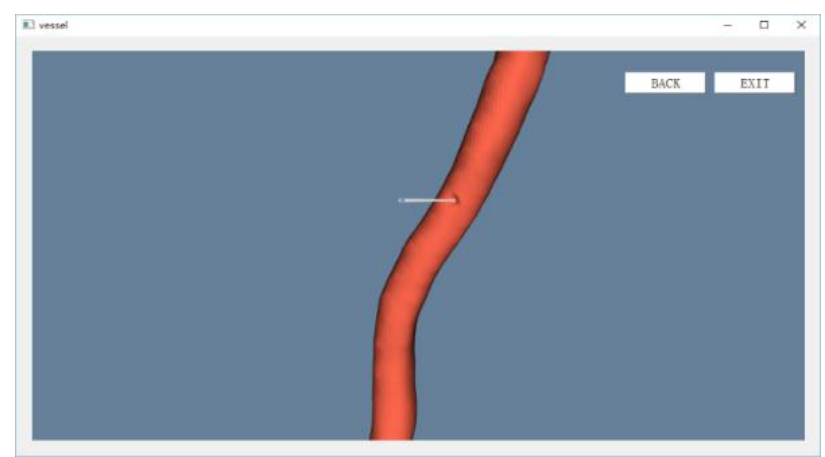

(a) Injection of needle

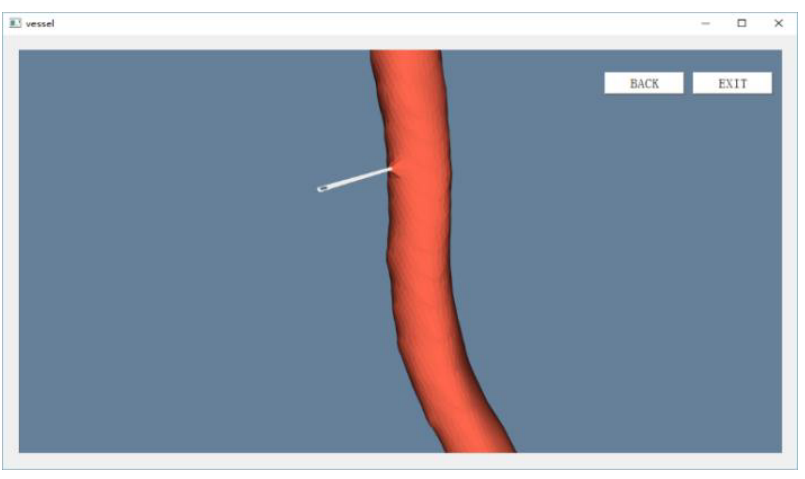

(b) Extraction of needle

Figure 4. Screenshots of intelligent platform

\section{Analysis and conclusion}

Mass-spring model have been developed greatly. The degree of simulation is improving, so the application fields are very wide. We focus on the surgery simulation based on Mass-spring and have realized an intelligent platform for vessel operation. There is still large space worth to explore and research.

In future work, we hope to solve three problems as follows.

1) Tuning parameters using Genetic Algorithm or Neural Network. Traditionally, the spring constants were set manually. It cannot satisfy the requirements for high precision and accuracy. So it is meaningful adopting machine learning algorithms to confirm those parameters;

2) $3 \mathrm{D}$ reconstruction for vessel model. As we all know, 3D model can provide intuitive knowledge for operators. However, the contrastographic picture of vessel is usually 2D graph [9]. How to reconstruct 3D vessel model based on contrastographic pictures from different angles? It will be an important problem.

3) Improving our intelligent platform by incorporating many different instruments simulation. Existing simulation 
software was limited one or few surgery instruments [10, 11]. But different instruments have different interaction performance when colliding with tissues. We need consider different situation to construct different models. Intelligent adapting to different medical tools will be our research target.

\section{Acknowledgment}

This research is supported by the National Natural Science Foundation of China (No.: 61202295), and the Technology Planning Project of Guangdong Province (No.: 2015A020209150), and the Technology Planning Project of Guangdong Province (No.: 2015A030401081) and College Students' Science and Technology Innovation Project of Guangdong Province (No.: 201510564283).

\section{References}

1. T. W. Sederberg. Free-form deformation of solid geometric models $[\mathrm{J}]$, Acm Siggraph Computer Graphics, 20(4):151-160 (2010)

2. S. F. Gibson.3D chainmail: a fast algorithm for deforming volumetric objects $[\mathrm{J}]$, Symposium on Interactive 3d Graphics, 149-154, 195(1997)

3. Louchet J., Provet X., Crochemore D.. Evolutionaly identification of cloth animation models[C]// computer Animation and Simulation '95 Maastricht: 44-54(1995)

4. W. Mollemans, F. Schutyser, J. V. Cleynenbreugel, P. Suetens. Tetrahedral Mass-spring Model for Fast Soft
Tissue Deformation[J], International Conference on Surgery Simulation \& Soft Tissue Modeling, 2673:145-154(2002)

5. Coutinho B. B., Oliveira A. A. F, Atencio Y. P., et al. Rainscene animation through particle systems and surface flow simulation by SPH [C] // 23th SIBGRAPIGramado, Brazil, 255-262(2010)

6. Thürey N., Wojtan C., Gross M., et al. A multiscale approach to mesh-based surface tension flows $[\mathrm{J}]$. ACM Transactions on Graphics, 29(4): 48-56(2010)

7. L.P. Nedel, D. Thalmann. Real time muscle deformation using mass-spring systems [J], Computer Graphics International(1998)

8. Iker A., Basil F. Filtering of high modal frequencies for stable real-time explicit integration of deformable objects using the Finite Element Method [J]. Progress in Biophysics and Molecular Biology, 103: 225-235 (2010)

9. Chris Guy D. F. An introduction to the principles of medical imaging [M]. London: Imperial college Press, 95-98(2005)

10. Sun S. H., Milan S. Lung segmentation refinement based on optimal surface finding utilizing a hybrid desktop virtual reality user interface $[\mathrm{J}]$. Computerized Graphics, 37:15-27(2013)

11. Lam C. K., Sundraj K. Virtual simulation of eyeball and extra ocular muscle reaction during cataract surgery [J]. Procedia Engineering, 41:150-155(2012) 